\title{
Analysis of hydrogen adsorption and surface binding configuration on tungsten using direct recoil spectrometry
}

\author{
R. D. Kolasinski*,(a), K. D. Hammond ${ }^{(\mathrm{b})}$, J. A. Whaley ${ }^{(\mathrm{a})}$, \\ D. A. Buchenauer ${ }^{(a)}$, and B. D. Wirth ${ }^{(\mathrm{b})}$ \\ ${ }^{(a)}$ Sandia National Laboratories, Hydrogen and Metallurgical Science Department, Livermore, \\ CA 94551 USA

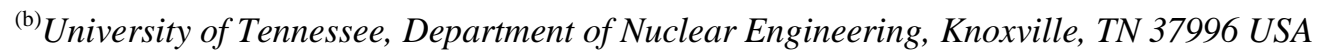

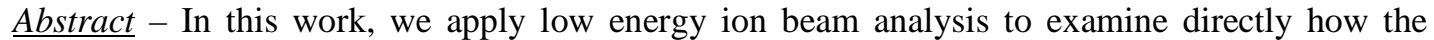
adsorbed hydrogen concentration and binding configuration on W(100) depend on temperature. We exposed the tungsten surface to fluxes of both atomic and molecular $\mathrm{H}$ and $\mathrm{D}$. We then probed the $\mathrm{H}$ isotopes adsorbed along different crystal directions using 1-2 $\mathrm{keV} \mathrm{Ne}{ }^{+}$ions. At saturation coverage, $\mathrm{H}$ occupies two-fold bridge sites on $\mathrm{W}(100)$ at $25{ }^{\circ} \mathrm{C}$. The $\mathrm{H}$ coverage dramatically changes the behavior of channeled ions, as does reconstruction of the surface $\mathrm{W}$ atoms. For the exposure conditions examined here, we find that surface sites remain populated with $\mathrm{H}$ until the surface temperature reaches $200{ }^{\circ} \mathrm{C}$. After this point, we observe $\mathrm{H}$ rapidly desorbing until only a residual concentration remains at $450{ }^{\circ} \mathrm{C}$. Development of an efficient atomistic model that accurately reproduces the experimental ion energy spectra and azimuthal variation of recoiled $\mathrm{H}$ is underway.
\end{abstract}

PACS codes: 68.43.Fg, 68.49.Sf, 34.50.-s, 61.85.+p

PSI-21 keywords: ion-surface collisions, hydrogen recycling, hydrogen adsorption, molecular dynamics simulations, tungsten

Corresponding author address (R. D. Kolasinski):

Sandia National Laboratories

P.O. Box 969, MS 9161

Livermore, CA 94550 USA

e-mail: rkolasi@ sandia.gov

Present affiliation (K. D. Hammond):

University of Missouri

Department of Chemical Engineering

Columbia, MO 65211 USA 


\section{Introduction}

Adsorption, recombination, and exchange of hydrogen isotopes on surfaces are of fundamental interest from the perspective of fuel recycling from plasma-facing materials in magnetic fusion devices. The atomic-scale details of these processes are difficult to resolve experimentally, largely because most surface analysis techniques cannot detect adsorbed hydrogen. Two exceptions include low energy ion scattering (LEIS) and direct recoil spectrometry (DRS), both of which provide direct information on the adsorbed hydrogen configuration on surfaces, as well as binding energies and exchange kinetics. Such insight complements ongoing fundamental atomic-scale modeling efforts (e.g. density functional theory or molecular dynamics) that focus on simulating the complex chemical effects that occur when surfaces are exposed to high flux plasmas. Experiments are needed to test these models, thereby motivating further refinements to interatomic potentials and other simulation inputs.

Both LEIS and DRS are forms of low energy ion beam analysis which provide both composition and structural information, with high surface-specificity $(<1 \mathrm{~nm}$ sampling depth.) In terms of the underlying physics, these techniques bear many similarities to their higher energy counterparts, Rutherford backscattering spectrometry and elastic recoil detection. We refer the reader to several excellent reviews $[1,2]$ for further details. In our prior work, we used LEIS and DRS to study the hydrogen binding configuration along open channels on the $\mathrm{W}(100)$ crystal plane [3]. While we were able to identify definitively two-fold bridge sites as the preferred binding site for complete coverage of the W surface with hydrogen, the details of the binding configuration at lower coverage were still unclear. Furthermore, the equilibrium surface coverage as a function of temperature was left unaddressed in our prior work. While this could be inferred from the $\mathrm{H}$ evolved from the surface during thermal desorption, DRS provides a direct measure. This coverage dependence is somewhat complicated due to reconstruction of the surface as the adsorbed $\mathrm{H}$ desorbs, where $\mathrm{W}$ surface atoms are displaced out of ideal bulk-terminated sites.

With the above concerns in mind, we address the temperature/coverage effects in greater detail in the present study. We use ion focusing along surface channels as a sensitive means of determining the 
hydrogen concentration present on the surface. To calibrate this process, we dosed the surface with molecular hydrogen $\left({ }^{1} \mathrm{H}\right)$ and deuterium $\left({ }^{2} \mathrm{H}\right)$, and measured the recoiled adsorbate signals as a function of temperature under a variety of conditions, with the eventual goal of comparing our results with recently-developed models of surface adsorption/recombination kinetics [4]. For comparison, we also performed a single set of measurements to study the adsorption of atomic D.

To complement our experimental results, we are developing a molecular dynamics (MD) model to simulate $\mathrm{H}$ migration on the $\mathrm{W}(100)$ surface. The use of MD allows for the inclusion of more sophisticated potentials and provides a more realistic calculation of interatomic forces than possible with the binary collision approximation models that are frequently used as inputs to recycling calculations. For the purpose of modeling the LEIS/DRS experiments, an MD framework to directly simulate $\mathrm{Ne}^{+}$scattering on the crystal surface is also under development.

\section{Experiment}

Our scattering experiments were performed using an ion energy spectrometer, which has been previously described in Ref. 5. Briefly, the system includes an ion source that produces massseparated beams through electron bombardment of inert gases. Scattered or recoiled particles are detected with a hemispherical analyzer (50 mm radius) with a $2^{\circ}$ acceptance angle. For this set of measurements, we used beams of low energy ( $1 \mathrm{keV}$ and $2 \mathrm{keV}) \mathrm{Ne}^{+}$ions to characterize a $7 \mathrm{~mm}$ diameter, $1 \mathrm{~mm}$ thick $\mathrm{W}$ single crystal (MaTecK $\mathrm{GmbH}$ ) that had been polished and aligned to within $0.1^{\circ}$ of the (001) plane. We secured the crystal with Ta wire along its outer circumference to a buttonstyle heater and an isolating sapphire disc. A well-ordered, clean sample surface could be achieved through repeated cycles of sputtering with $2 \mathrm{keV} \mathrm{Ne}^{+}$ions at grazing incidence followed by heating to $450{ }^{\circ} \mathrm{C}$. The $\mathrm{Ne}^{+}$ion beam ( $100 \mathrm{nA}$ current) could be focused to a $0.5 \mathrm{~mm}$ spot, which was then rastered over a $2 \mathrm{~mm} \times 2 \mathrm{~mm}$ area, producing an incident flux of $1.5 \times 10^{13} \mathrm{~cm}^{-2} \mathrm{~s}^{-1}$. The raster amplitude was adjusted with incidence angle to maintain a constant spot size and flux on the sample surface. To verify that the low flux from the $\mathrm{Ne}^{+}$analysis beam did not damage the surface, we frequently compared with a set of measurements that were performed shortly after the pristine crystal 
was inserted into our vacuum system. The scans were identical, suggesting that the beam had a negligible effect on the surface structure.

The concentration of hydrogen isotopes on the W crystal surface could be controlled by varying the surface temperature or the incident flux of hydrogen isotopes. For the purposes of dosing, we introduced the hydrogen through a tungsten capillary ( $1 \mathrm{~mm}$ inner diameter) into our vacuum system. The tungsten capillary could be heated by electron bombardment to $1700{ }^{\circ} \mathrm{C}$ to assure $100 \%$ dissociation of the effusing hydrogen flux [6], thus allowing us to dose with either atomic or molecular species. The atomic state of the flux from our doser was confirmed by examining D adsorption on an $\mathrm{Al}(100)$ single crystal cooled with liquid nitrogen to $-160{ }^{\circ} \mathrm{C}$. In contrast to $\mathrm{W}$, prior measurements indicate a very low sticking coefficient $\left(<10^{-4}\right)$ for $\mathrm{D}_{2}$ on $\mathrm{Al}(100)$, whereas atomic $\mathrm{D}$ has a high sticking coefficient $(0.6 \pm 0.1)$ [7]. Exposure of our Al crystal to atomic D rapidly increased the height of the associated recoil peak, whereas $\mathrm{D}_{2}$ exposure produced no observable difference.

The key geometrical parameters for this set of experiments are illustrated in Fig. 1(a), including the angle of incidence of the analysis beam $(\alpha)$, the azimuth on the W(100) surface $(\phi)$, and observation angle $(\theta)$. Recall that the energies of the scattered and recoiled particles are used to identify species present on the surface. Fig. 1(b) shows ion energy spectra for $2 \mathrm{keV} \mathrm{Ne}+\rightarrow \mathrm{W}(100)$ in cases where the surface is exposed to background gases present within the vacuum system only as well as during exposure to $\mathrm{D}_{2}(\mathrm{~g})$. Note that we have normalized all energies relative to that of the incident beam $\left(E_{0}\right)$. Within the two spectra, three prominent peaks are readily visible, corresponding to $\mathrm{H}, \mathrm{D}$, and $\mathrm{W}$ present on the surface. The vertical lines indicate the theoretical positions for these peaks, calculated using classical kinematics and ignoring any inelastic energy loss mechanisms. Based on calculations using the binary collision code MARLOWE [8], the smaller peak centered at $E / E_{0} \approx 0.75$ is attributed to a more complex scattering process involving multiple collisions of $\mathrm{Ne}^{+}$ ions with the $\mathrm{H}$ layer and $\mathrm{W}$ substrate. Even when the surface is not dosed, a peak associated with recoiled $\mathrm{H}$ is still present, which we attribute to dissociative chemisorption of residual $\mathrm{H}_{2}(\mathrm{~g})$ in our vacuum system. Hydrogen dissolved within the $\mathrm{W}$ lattice may also diffuse to the surface, although 
this is expected to be only a minor contribution to the $\mathrm{H}$ surface concentration at room temperature. Using $\mathrm{D}_{2}$ as the exposure gas helps to circumvent this issue.

Once the surface was exposed to $\mathrm{D}_{2}(\mathrm{~g})$, the $\mathrm{H}$ signal diminished rapidly and the $\mathrm{W}$ signal decreased slightly. We systematically tested different molecular fluxes to the surface, finding that saturation coverage at $25{ }^{\circ} \mathrm{C}$ could be assured with a partial pressure of $3.0 \times 10^{-7}$ Torr. This concentration depends on a dynamic equilibrium dictated by the arrival rate of $\mathrm{H}_{2}(\mathrm{~g})$, the sticking and reflection coefficients, as well as the desorption rate due to recombination. One key concern is that the incident ion beam used for our measurements also contributes to desorption. To minimize this effect, we ensured that the exposure flux produced an arrival rate of $\mathrm{H}_{2}(\mathrm{~g})$ at the surface that much higher than that of the incident $\mathrm{Ne}^{+}$ions. Based on the kinetic theory of gases, the aforementioned partial pressure of $3.0 \times 10^{-7}$ Torr corresponds to a flux of $3.0 \times 10^{14} \mathrm{D}_{2} \mathrm{~cm}^{-2} \mathrm{~s}^{-1}$, or a factor of 20 higher than the ion arrival rate at the surface. In practice, because the angular distribution of emitted particles is highly peaked along the capillary centerline, the arrival rate of $D$ at the sample surface is much higher than estimated above.

We were able to calculate the chemisorbed concentrations of $\mathrm{H}$ and $\mathrm{D}$ by integrating the area under each peak and correcting the result for the recoil cross-sections for each species. For $2 \mathrm{keV} \mathrm{Ne}{ }^{+}$ and a laboratory recoil angle of $\theta=25^{\circ}$, the relevant cross-sections are: $5.93 \times 10^{-2} \AA^{2} / \mathrm{sr}(\mathrm{H})$ and $2.96 \times 10^{-2} \AA^{2} / s r$ (D). These values were calculated using the Biersack-Haggmark approximation for the scattering integrals and assuming a ZBL interatomic potential [9]. Assuming that the dosed case shown in Fig. 1(b) corresponds to saturation, the $\mathrm{H}$ coverage is 6\%, with the remaining D comprising $94 \%$ of the total. Note that this analysis assumes that the surface H and D occupy the same adsorption sites.

\section{Coverage as a function of temperature}

To understand the coverage dependence as a function of temperature better, we performed a systematic series of controlled adsorption experiments, in a manner similar to the procedures specified in Ref. 4. This involved rapidly heating the crystal to $450{ }^{\circ} \mathrm{C}$ while exposing the sample to different 
141 fluxes of deuterium or protium. In each case, the sample was held at $450{ }^{\circ} \mathrm{C}$ for $30 \mathrm{~min}$ and then

142 allowed to cool at a linear rate of $7.1^{\circ} \mathrm{C} / \mathrm{min}$. (This corresponds to a $1 \mathrm{hr}$ cooling time from $450{ }^{\circ} \mathrm{C}$ to

143 room temperature.) The observed concentrations during adsorption were found to be independent of

144 the cooling rate up to $14.2^{\circ} \mathrm{C} / \mathrm{min}$. During the entire process, we monitored the recoiled peak height

145 corresponding to hydrogen isotopes adsorbed on the surface. We obtained all measurements using a 2

$146 \mathrm{keV} \mathrm{Ne}^{+}$beam at an incidence angle of $\alpha=80^{\circ}$ to probe the hydrogen present along $\langle 100\rangle$ surface

147 directions.

148 In Fig. 2, we show recoil signals from D adsorbed on the W(100) surface as the temperature is

149 slowly cooled from $450{ }^{\circ} \mathrm{C}$ using the procedure described above. In case (a), three different D

150 exposure pressures are considered, ranging over an order of magnitude between $7.4 \times 10^{-8}$ Torr to

$1511.1 \times 10^{-6}$ Torr. To aid in the interpretation of the data, we rendered the adsorption isobars as Arrhenius

152 plots. In addition, we assumed that once the surface temperature declined below $100{ }^{\circ} \mathrm{C}$, the surface

153 was saturated with D, hence the isobars were normalized to this saturation condition. Keep in mind

154 that the sampling depth of our analysis beam at oblique incidence is $<1 \mathrm{~nm}$, hence all signals shown

155 here correspond to D present on the surface. Regardless of the exposure pressure, the same basic

156 adsorption curve shape is evident. In the Arrhenius coordinates, the quantity adsorbed follows a

157 nearly linear dependence at temperatures greater than $300^{\circ} \mathrm{C}$. Afterward, the slope of the isobar

158 decreases until it reaches a constant level for $T<150{ }^{\circ} \mathrm{C}$. As shown in case (a), modifying the

159 exposure pressure offsets the adsorption profile along the $1 / T$ axis without affecting its slope.

160 It was also of interest to determine whether dosing the samples with atomic $\mathrm{D}$ versus $\mathrm{D}_{2}$ would

161 produce any appreciable difference in the quantity adsorbed on the surface. Adsorption curves for

162 both cases are shown in Fig. 2(b). We could not discern an appreciable difference within the error of

163 our experiment. We also noted a slight offset between chemisorption of $\mathrm{H}_{2}$ vs. $\mathrm{D}_{2}$ on the surface, but

164 the difference was very slight and within the experimental error. 


\section{Variation of binding configuration as a function of temperature}

For single crystal surfaces, it is possible to use LEIS and DRS to gain detailed information about the surface structure. The main effect exploited here is surface channeling. At grazing angles of incidence, the energy of the incoming $\mathrm{Ne}^{+}$particles perpendicular to the sample surface is very small, enabling the tungsten substrate atoms to gently deflect them along open channels on the crystal surface. For $\mathrm{W}(100)$, the predominant channels are along $\langle 100\rangle$ and $\langle 110\rangle$ directions, as illustrated by the shaded regions in Fig. 3. Ion focusing increases the likelihood of collisions between the incident $\mathrm{Ne}^{+}$and any adsorbed hydrogen isotopes bound along a given channel. Hence, enhanced signals from recoiled hydrogen would be observed along surface channels containing hydrogen, thereby enabling a definitive identification of the binding configuration. More details on the use of surface channeling to study adsorbed $\mathrm{H}$ may be found in Ref. 3 .

To aid in our analysis of the adsorbed $\mathrm{H}$ layer, we monitored the recoiled $\mathrm{H}$ peak height over a wide range of incidence angles $(\alpha)$ and azimuths $(\phi)$. These data are compiled into the maps depicted in Fig. 4. To examine the binding configuration as a function of temperature (and surface $\mathrm{H}$ coverage), we considered four different temperatures between $25^{\circ} \mathrm{C}$ and $350{ }^{\circ} \mathrm{C}$, as shown in cases (a-d). All of the maps were acquired at an observation angle of $\theta=45^{\circ}$ using $1 \mathrm{keV} \mathrm{Ne}{ }^{+}$; in each case we exposed the surface to a $\mathrm{H}_{2}$ partial pressure of $3 \times 10^{-7}$ Torr. Immediately evident in each map is the presence of distinct hydrogen recoil structures along $\langle 100\rangle$ and $\langle 110\rangle$ directions that repeat every $90^{\circ}$, annotated respectively by "A" and "B" in Fig. 4. The slight variability in the repeating pattern with azimuth is due to a small misalignment of the crystal surface normal with respect to the incident beam.

The saturation coverage case at $25^{\circ} \mathrm{C}$ depicted in Fig. 4(a) is nearly identical to our prior observations [3], with the same distinct hydrogen recoil patterns aligned along the $\langle 100\rangle$ and $\langle 110\rangle$ directions. This pattern provides clear evidence of adsorbed $\mathrm{H}$ residing in 2-fold (bridge) sites on the $\mathrm{W}(100)$ surface at $25^{\circ} \mathrm{C}$ at saturation coverage. Had the hydrogen been residing in other high symmetry surface sites (such as four-fold hollow or top), local maxima in recoiled hydrogen intensity would not be observed along both the $\langle 100\rangle$ and $<110\rangle$ azimuths. In addition, prior low energy electron diffraction (LEED) measurements provide compelling evidence that at $25{ }^{\circ} \mathrm{C}$, the $\mathrm{W}$ surface 
atoms on the (100) plane reside in a $1 \times 1$ configuration when saturated with hydrogen [10,11]. (Note that this notation indicates the basic translation vectors that describe the surface superstructure.) This configuration is illustrated in Fig. 3(a), and corresponds to $2 \mathrm{H} / \mathrm{W}$ (or $2 \times 10^{15} \mathrm{H} / \mathrm{cm}^{2}$ ). Note that the dashed line in this figure indicates the surface unit cell.

Our previous analysis [3] did not reveal the definitive binding configuration at partial coverage. In the present study, the carefully controlled $\mathrm{H}$ dosing and systematic examination of coverage with temperature provide an opportunity for further analysis. While the relative positions of the adsorbed $\mathrm{H}$ cannot be detected directly with LEED, such measurements provide valuable insight into the W(100) substrate configuration at different hydrogen isotope coverages [10,11]. At room temperature, the clean $\mathrm{W}(100)$ surface is in a simple $1 \times 1$ configuration, with the $\mathrm{W}$ atoms residing close to their bulk lattice sites (aside from a small relaxation between the first few atomic layers.) As hydrogen isotopes adsorb, the substrate reconstructs to form a $c(2 \times 2)+\mathrm{H}$ structure. Here the $\mathrm{W}$ surface atoms displace laterally by $0.2 \AA$ toward the hydrogen adatoms, as depicted in Fig. 3(b). Finally, as the surface approaches saturation, it reverts back to a $1 \times 1$ phase, as shown in Fig. 3(a).

The positions of the tungsten surface atoms are crucial, as they affect both the adsorbed hydrogen configuration as well as how ions are scattered along the surface. In the reconstructed configuration, the surface channels along <100> directions narrow by $0.4 \AA$ (by $13 \%$ ). At $150{ }^{\circ} \mathrm{C}$, the intensity patterns along $\langle 100\rangle$ and $\langle 110\rangle$ directions still persist, indicating that bridge sites are still preferred, even at somewhat lower coverage. The $\mathrm{H}$ recoil patterns, however, subtend a smaller azimuthal range than is evident in the saturation coverage case. This behavior is consistent with narrowing of the surface channels on the reconstructed surface. At $250^{\circ} \mathrm{C}$ and higher, the same basic recoil pattern persists, thereby confirming that $\mathrm{H}$ remains in bridge sites even at low coverage.

\section{Future Model Development}

While the analysis discussed here provides support for bridge site occupation for all levels of $\mathrm{H}$ coverage on $\mathrm{W}(100)$, a more compelling case can be made through computational modeling of surface collisions. Because grazing incidence angles are used to probe the tungsten surface, the incident ions 
interact with many surface atoms at once. In this sense, MD is a better computational approach to

219 simulate this than binary collision models. While MD simulations are often considered impractical for

220 simulating scattering, simplifying assumptions (as discussed by Danailov et al. [12]) can be

incorporated to make the problem more tractable. We are developing simulations of ion scattering at

grazing incidence along $\langle 100\rangle$ and $<110\rangle$ surface channels that involve varying the amount of $\mathrm{H}$

present to match the measured surface coverage.

A key starting point for this work involves the evaluation of interatomic potentials for

incorporation into MD simulations. Since most potentials are optimized to reproduce bulk properties,

surface properties may not be well represented. Such problems aside, we do not foresee any

insurmountable obstacles to applying MD techniques to the scattering analyses presented here. The coupling of such experiments and models provides a promising avenue for simulating atomic-scale phenomena of interest to ITER and other plasma devices, and will be presented in future publications.

\section{Acknowledgments}

231

232

233

We thank Thomas Felter and Robert Bastasz for helpful discussions regarding this work. Sandia National Laboratories is a multi-program laboratory managed and operated by Sandia Corporation, a wholly owned subsidiary of Lockheed Martin Corporation, for the U.S. Department of Energy's National Nuclear Security Administration under contract DE-AC04-94AL85000. The contributions of KDH and BDW are supported by the Plasma-Surface Interactions Science Center funded by the U.S. Department of Energy, Office of Fusion Energy Sciences under award number DE-SC00-02060.

\section{References}

[1] R. Bastasz and W. Eckstein, Particle Scattering, in: E. N. Kaufmann (Ed.), Characterization of Materials, John Wiley \& Sons, Inc., 2012, 1-16.

[2] H. Niehus, W. Heiland, and E. Taglauer, Surf. Sci. Rep., 17 (1993) 213.

[3] R. D. Kolasinski, N. C. Bartelt, J. A. Whaley, and T. E. Felter, Phys. Rev. B, 85 (2012) 115422. 
242 [4] S. Markelj, O. V. Ogorodnikova, P. Pelicon, T. Schwarz-Selinger, and I. Čadež, Appl. Surf. Sci., $243 \quad 282(2013) 478$.

244 [5] R. Bastasz, J. A. Whaley, T. A. Lograsso, and C. J. Jenks, Philos. Mag., 86 (2006) 855.

245 [6] C. Eibl, G. Lackner, and A. Winkler, J. Vac. Sci. Technol. A 16 (1998) 2979.

246 [7] A. Winkler, Appl. Phys. A 67 (1998) 637.

247 [8] M. T. Robinson, Phys. Rev. B 40 (1989) 10717.

248 [9] J. F. Ziegler, J. P. Biersack, U. Littmark, The Stopping and Range of Ions in Solids: Volume 1, 249 Pergamon Press, New York, 1985.

250 [10] R. A. Barker and P. J. Estrup, Phys. Rev. Lett. 41 (1978) 1307.

251 [11] D. A. King, Phys. Scr. T4 (1983) 34.

252 [12] D. Danailov, D. J. O'Connor, K. J. Snowdon, Surf. Sci. 347 (1996) 215. 


\section{Figure Captions}

254 FIG. 1: (a) Illustration of the geometry of the scattering experiments showing definitions of the polar

$255(\alpha)$, azimuthal $(\phi)$, and observation $(\theta)$ angles. (b) Ion energy spectra for scattered $\mathrm{Ne}$ and recoiled $\mathrm{H}$

256 and D from the W(100) surface. Dashed lines correspond to calculated elastic scattering and recoil

257 energies for the indicated surface species.

258 FIG. 2: Adsorption profiles for hydrogen isotopes on W(100). Case (a) shows the dependence on $\mathrm{D}_{2}(\mathrm{~g})$

259 exposure pressure, whereas case (b) compares adsorption of different isotopes and illustrates the

260 effect of dosing with atomic species.

261 FIG. 3: Hydrogen configuration on W(100) at (a) saturation coverage, and (b) partial coverage. Large

262 markers indicate W surface atoms, small markers indicate adsorbed H. Prominent channeling

263 directions are indicate by the shaded regions, and the surface unit cell is indicated by the dashed line.

264 FIG. 4: Ion scattering maps depicting H recoiled from the W(100) surface, for four different surface

265 temperatures. Experimental conditions: $\mathrm{H}_{2}(\mathrm{~g})$ partial pressure of $3 \times 10^{-7}$ Torr; $\theta=45^{\circ}$. 


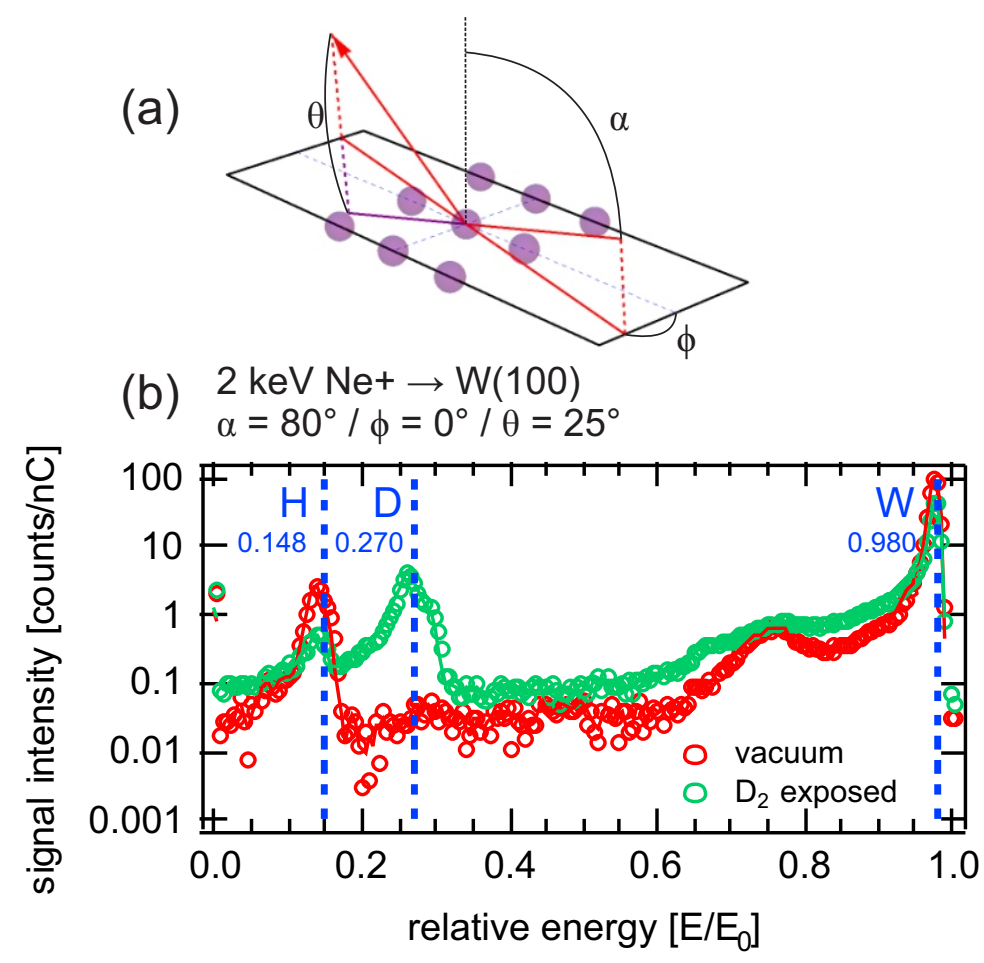

Fig. 1 


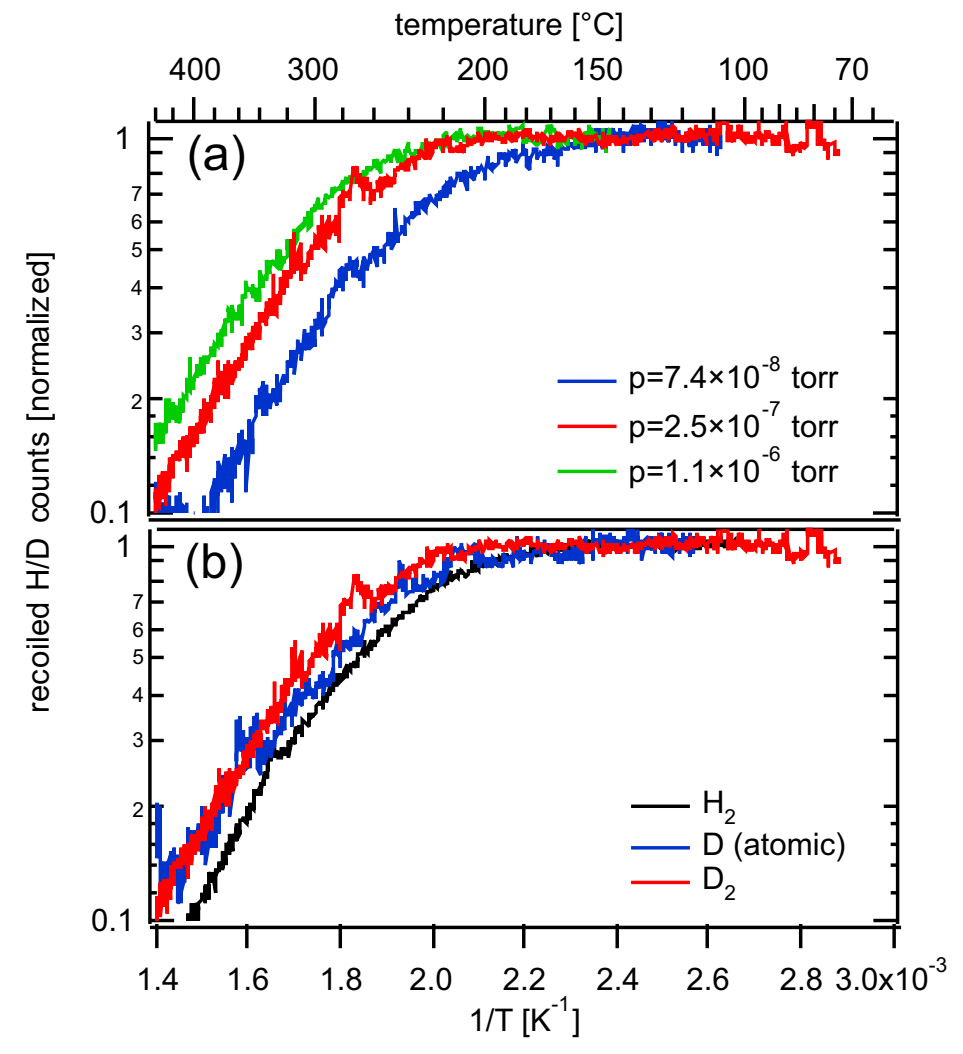

Fig. 2 

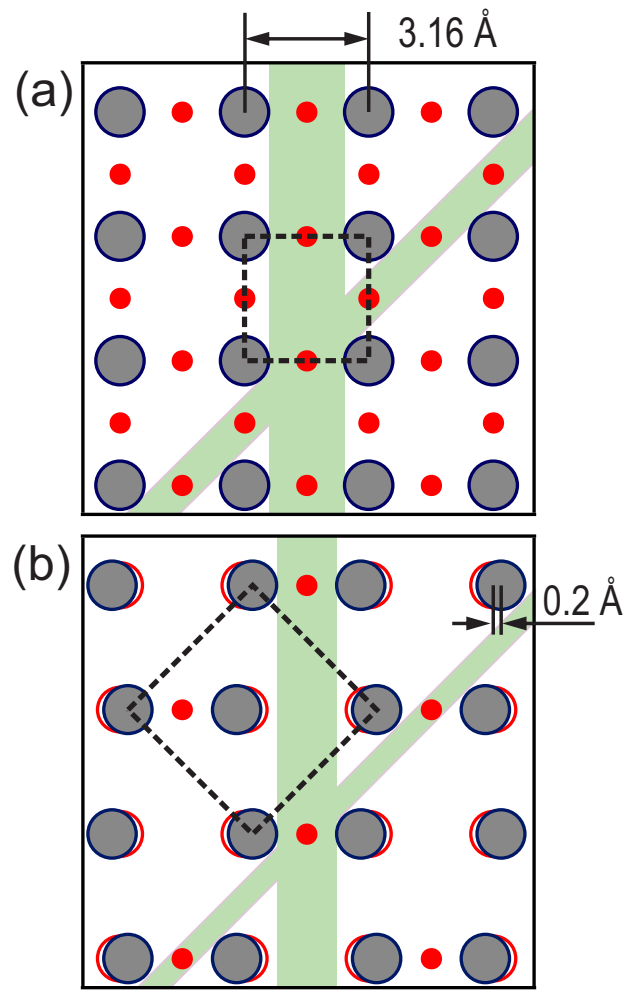

Fig. 3 


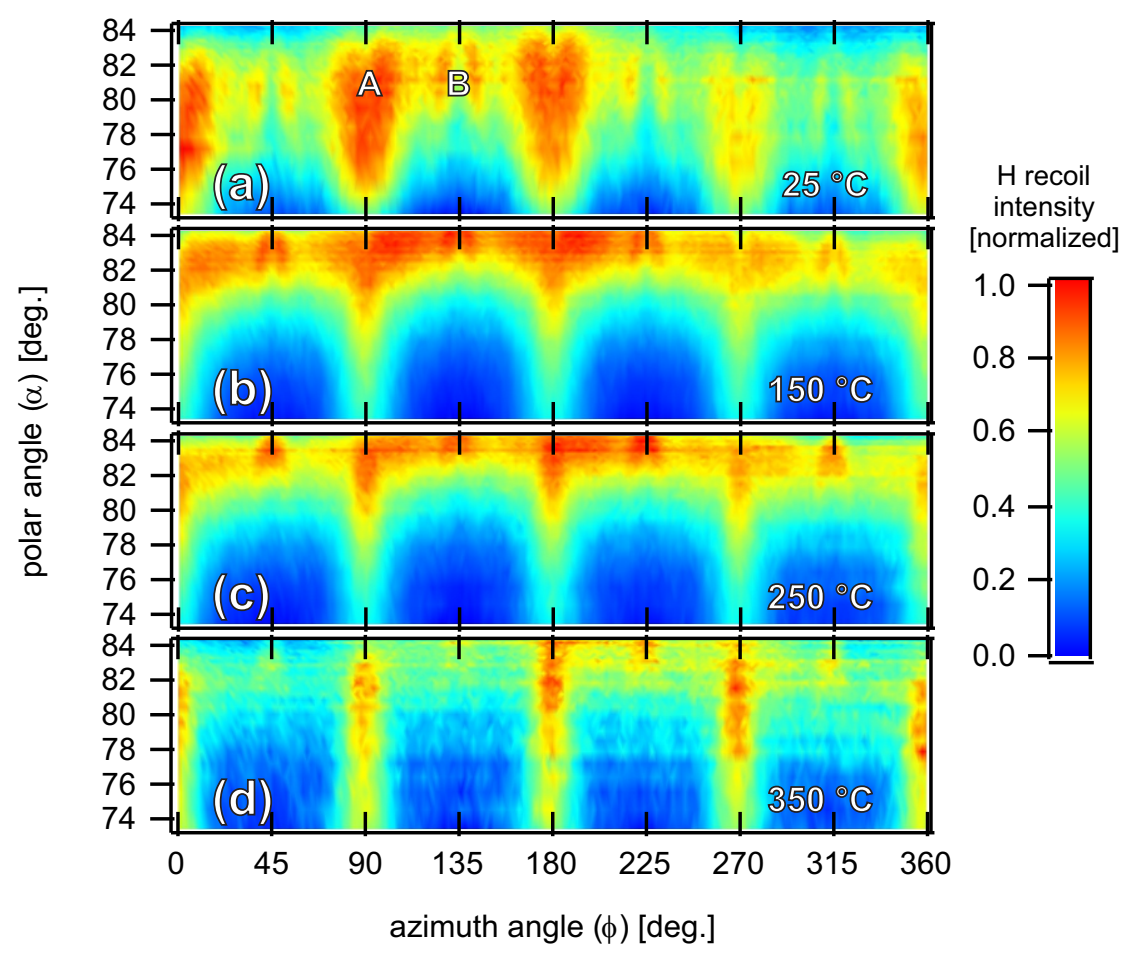

Fig. 4 